\title{
In-Operando Optical Imaging of Temporal and Spatial Distribution of Polysulfides in Lithium-Sulfur Batteries
}

Yongming Sun ${ }^{\mathrm{a}, 1}$, Zhi Wei Seh ${ }^{\mathrm{a}, 1}$, Weiyang Li ${ }^{\mathrm{a}}$, Hongbin $\mathrm{Yao}^{\mathrm{a}}$, Guangyuan Zheng ${ }^{\mathrm{b}}$, Yi

$$
\mathrm{Cui}^{\mathrm{a}, \mathrm{c}, *}
$$

${ }^{a}$ Department of Materials Science and Engineering, Stanford University, Stanford, California 94305, USA

${ }^{b}$ Department of Chemical Engineering, Stanford University, Stanford, California 94305, USA

${ }^{c}$ Stanford Institute for Materials and Energy Science, SLAC National Accelerator Laboratory, Menlo Park, California 94025, USA

*Correspondence to: yicui@stanford.edu (Yi Cui)

${ }^{1}$ These authors contributed equally to this work.

\begin{abstract}
Understanding the behavior of soluble intermediate lithium polysulfide species is vitally important for improving the electrochemical performances of lithium-sulfur batteries. Herein we explore a simple in-operando lithium-sulfur cell design to enable direct visualization of the formation of the soluble polysulfide species and their temporal and spatial distribution over the entire discharge/charge cycle under an optical microscope. Our results reveal detailed evidence of electrochemical degradation in lithium-sulfur batteries and help us to understand the improvements in electrochemical performances using advanced lithium-sulfur cell designs. As examples, we show that a cathode consisting of hollow sulfur nanoparticles with a conductive polymer poly(3,4-ethylenedioxythiophene) (PEDOT) coating exhibits
\end{abstract}


significantly reduced dissolution of polysulfides into the electrolyte, and thus superior electrochemical performance could be achieved. Moreover, the trapping of soluble polysulfide species in the cathode side was also confirmed in our designed in-operando lithium-sulfur cell with a Nafion modified separator.

Keywords: In-operando lithium-sulfur cell, Direct visualization, Polysulfides, Temporal and spatial distribution

\section{Introduction}

Lithium-sulfur is an attractive battery chemistry to meet the increased demand from portable electronics, electrical transportation and large-scale stationary energy storage due to its high specific density (2600 Wh/kg), high natural abundance of sulfur, and low cost potential [1]. However, several issues still remain to make it a viable technology. A critical problem is the quick capacity decay upon cycling due to the dissolution of the intermediates (lithium polysulfides, $\mathrm{Li}_{2} \mathrm{~S}_{x}, 4 \leqslant x \leqslant 8$ ) into the electrolyte and the "shuttle effect" of these soluble species [2-5]. To overcome these problems, extensive research has been conducted with a focus on engineering the electrode structure and composition [2]. To date, several important strategies, including nanoporous carbon-sulfur composites [6-9], graphene (oxide)sulfur composites [10-12], conductive polymer-sulfur composites [13,14], as well as oxide coating/composites [15], have been explored to encapsulate polysulfides to suppress their dissolution during cycling. In our group, we also demonstrated rational internal hollow space design such as hollow carbon encapsulated sulfur [16,17], sulfur- $\mathrm{TiO}_{2}$ yolk-shell nanoparticles [18] and polymer-encapsulated hollow sulfur particles [19,20]. Despite these exciting progress, there still exists appreciable polysulfide dissolution (10-20\%) in the electrolyte as measured by inductively coupled plasma-optical emission spectroscopy (ICPOES) [18-20]. 
Recently there have also been several reports taking the direction of increasing the utilization of soluble polysulfide species, including spatial control deposition of polysulfides on patterned electrodes [21], insertion of a microporous carbon paper between the cathode and separator [22], modification of separators (e.g., separators coated with a Nafion film [23] and a graphene membrane [24]). Moreover, a breakthrough has been achieved in the electrolytes for lithium-sulfur batteries. It is found that the dissolution of lithium polysulphide can be inhibited by using a "solvent-in-salt" electrolyte with ultrahigh salt concentration and high lithium-ion transference number [25]. These works demonstrate improved cycling performance and represent exciting progress as well.

For all the above studies, it is vitally important to understand the behavior of polysulfide species in lithium-sulfur batteries. To this end, a great deal of work has been done to examine sulfur cathodes and polysulfides using ex-situ techniques, including Raman, Fourier transform infrared spectroscopy (FTIR), X-ray photoelectron spectra (XPS) and X-ray diffraction (XRD) [26-28]. Very recently, in-operando Raman [29] and in-operando X-ray diffraction and imaging [30] investigations have been carried out, providing valuable information. Despite all the above achievements, a direct visualization and full understanding of the spatial and temporal distribution of polysulfides in lithium-sulfur batteries is still lacking.

Here we demonstrate a simple in-operando lithium-sulfur cell design, which allows us to directly visualize the spatial and temporal distribution of lithium polysulfide species over the entire charge/discharge cycle under an optical microscope. Because of this capability, this inoperando technique, affords an excellent diagnostic tool to determine quickly the effect of polysulfide encapsulation in sulfur cathodes and trapping with modified separators. As examples, the effect of encapsulation of hollow sulfur nanoparticles with a conductive polymer poly(3,4-ethylenedioxythiophene) (PEDOT) was confirmed by our technique. The localization of polysulfides was also directly shown with a Nafion-modified separator. 


\section{Experimental}

\section{The in-operando lithium-sulfur cell design}

Figure 1a shows the schematic of an in-operando lithium-sulfur cell. In our design, a small glass pipette with its tip cut off was used for housing the in-operando cell (Figure 1b). Stainless steel bolts with circular cross sections (Figure 1c, left) were used as the current collectors and mechanical supports of the sulfur cathode (Figure 1c, right) and lithium anode (Figure 1d). The Celgard 2300 membrane was used as the separator (Figure 1e, left). A glass microfiber filter (GF/A, Watman, Figure 1e right) was inserted between the anode and the separator, which can buffer the mechanical shock to protect the electrodes during their assembly, adsorb and localize the electrolyte. The Celgard 2300 membrane is too thin $(\sim 25$ $\mu \mathrm{m})$ for focus and observation under an optical microscope and here the glass microfiber filter with the thickness of $\sim 0.5 \mathrm{~mm}$ functions as a window to observe the color change of the electrolyte during the entire charge/discharge cycle. The electrolyte was a solution of $1 \mathrm{M}$ lithium bis(trifluoromethanesulfonyl)imide in 1:1 (volume ratio) 1,2-dimethoxyethane and 1,3-dioxolane containing $1 \mathrm{wt} \% \mathrm{LiNO}_{3}$. The in-operando cell was sealed with silver conductive epoxy after its assembly (Figure 1f).

\section{Preparation of pristine sulfur and sulfur-PEDOT composite electrodes and lithium counter electrodes}

The pristine sulfur electrodes were prepared by mixing the sublimed sulfur powder (99.98\%, ALDRICH), acetylene black (Super-P) and polyvinylidene fluoride (PVDF) in a weight ratio of 60:30:10 in N-methyl-2-pyrrolidinone (NMP). The sulfur-PEDOT composite was prepared according to our previous report [20]. The slurry for PEDOT-encapsulated hollow sulfur nanoparticles comprised of $70 \mathrm{wt} \%$ sulfur-PEDOT composite, $20 \mathrm{wt} \%$ super-P and $10 \mathrm{wt} \%$ PVDF in NMP solvent. After the slurries were dropped onto the smooth end of stainless steel bolts (with diameter of $\sim 3.5 \mathrm{~mm}$ ), the as-prepared sulfur-based electrodes were 
dried at $60{ }^{\circ} \mathrm{C}$ in vacuum. The mass loading of the active sulfur was $\sim 0.2 \mathrm{mg} / \mathrm{cm}^{2}$ in the asprepared electrodes. A lithium foil was attached onto the end of the steel bolt which was used as the counter electrode in the in-operando cells.

\section{Preparation of functional separators with Nafion coating}

A Celgard 2300 membrane was used as the cell separator. For the preparation of polysulfide-blocking functional separators, a LIQUION solution (Nafion in a mixture of water and alcohols, LQ-1105-1100 EW at $5 \mathrm{wt} \%$, Ion Power) was directly dropped onto a Celgard 2300 membrane and dried in the fume hood.

\section{Assembly and electrochemical measurements of the in-operando lithium-sulfur cells}

The in-operando cells were assembled in an argon-filled glove box. Typically, a sulfur electrode was firstly encapsulated in the thin glass tube using silver conductive epoxy. After the silver conductive epoxy solidified, a separator was put onto the sulfur-based electrode, followed by a glass microfiber filter. Then $5 \mu \mathrm{L}$ of electrolytes was dropped onto the glass microfiber filter. Finally, the as-made lithium counter electrode was sealed in the glass tube from the opposite side of the cathode with silver conductive epoxy. Galvanostatic charge/discharge test was performed using a single channel potentiostat/galvanostat (SP-50, EC-Lab Electrochemical Measurement Instruments) at the current densities of $10 \mu \mathrm{A}$ with a fixed potential range of $1.7-2.6 \mathrm{~V}$ vs. $\mathrm{Li}^{+} / \mathrm{Li}$ at room temperature. The low current was utilized to maximize the degree of electrode reactions during the discharge/charge processes. Instead of a sloping high voltage plateau (2.6-2.1 V) usually observed in previous works, a flatter plateau was observed in our lithium-sulfur potential profile due to the much lower current density used [2-5]. Digital and optical microscopy images were taken synchronously. The in-operando optical imaging were carried out in the region of glass filters. The color intensity of the electrolyte is proportional to the concentration of polysulfides, which is used as the basis for determining the amount of polysulfides. Similar to the previous studies of 
spectra [31], colors of the electrolyte could only be interpreted in terms of an average stoichiometry of the dissolved polysulfides. The differences among various polysulfides were omitted.

\section{Results and discussion}

An in-operando cell with a pristine sulfur electrode was cycled at a current of $10 \mu \mathrm{A}$ in the potential range of 1.7-2.6 V vs. $\mathrm{Li}^{+} / \mathrm{Li}$. Optical and digital images were taken simultaneously to record the color change at various cell potentials during the discharge/charge processes. Figure 2 a shows the typical discharge curve of the as-made in-operando cell during the first cycle. At the high voltage plateau (2.6-2.1 V), soluble polysulfide species, including $\mathrm{S}_{8}{ }^{2-}, \mathrm{S}_{6}{ }^{2-}$, and $\mathrm{S}_{4}{ }^{2-}$, are produced $\left(\mathrm{S}_{8}+2 \mathrm{e}^{-} \rightarrow \mathrm{S}_{8}{ }^{2-}, 3 \mathrm{~S}_{8}{ }^{2-}+2 \mathrm{e}^{-} \rightarrow 4 \mathrm{~S}_{6}{ }^{2-}, 2 \mathrm{~S}_{6}{ }^{2-}+2 \mathrm{e}^{-} \rightarrow\right.$ $3 \mathrm{~S}_{4}{ }^{2-}$ ) [2-5]. Subsequently, upon further discharge, a low voltage plateau at $2.1 \mathrm{~V}$ was observed, which corresponds to the formation of insoluble $\mathrm{Li}_{2} \mathrm{~S}_{2}$ and $\mathrm{Li}_{2} \mathrm{~S}\left(\mathrm{~S}_{4}{ }^{2-}+4 \mathrm{Li}^{+}+2 \mathrm{e}^{-}\right.$ $\rightarrow 2 \mathrm{Li}_{2} \mathrm{~S}_{2}, 2 \mathrm{Li}_{2} \mathrm{~S}_{2}+2 \mathrm{Li}^{+}+2 \mathrm{e}^{-} \rightarrow 2 \mathrm{Li}_{2} \mathrm{~S}$ ) [2-5]. The inset of Figure 2a and Figure $\mathrm{S} 1$ compare the colors of the electrolyte during the initial discharge process and Figure $2 \mathrm{c}$ shows the corresponding change of gray level. The evolution of colors and its consistency with the electrochemical profiles allow us to propose the temporal distribution of polysulfides over the discharge process. We see that during discharge at the high voltage plateau (2.6-2.1 V), the electrolyte gradually becomes darker from colorless to gray to brown-yellow, as shown by an increasing gray level as well. At the end of the high voltage plateau $(2.1 \mathrm{~V})$, corresponding to the depth of discharge (DOD) of $\sim 38.9 \%$, the electrolyte exhibits the darkest color and highest gray level (Figure 2c), indicating the maximum concentration of these soluble polysulfides and the end of the $S_{8}$ consumption. This result is consistent with previous studies by Operando X-ray absorption spectroscopy [32] and electrochemical impdance spectroscopy [33]. Subsequently, upon further discharge, the electrolyte becomes lighter in color and the 
gray level decreases (Figure 2c). This is due to the conversion of soluble polysulfide species $\left(\mathrm{S}_{8}{ }^{2-}, \mathrm{S}_{6}{ }^{2-}\right.$, and $\left.\mathrm{S}_{4}{ }^{2}\right)$ into insoluble $\mathrm{Li}_{2} \mathrm{~S}_{2}$ and $\mathrm{Li}_{2} \mathrm{~S}$ species which precipitate back onto the cathode surface. In our in-operando lithium-sulfur cell, we see that the electrolyte still remains light yellow (instead of colorless) at the end of discharge, indicating that some soluble polysulfides still remain in it. This loss of active material explains why the discharge capacities of sulfur cathodes in most reports are always less than of the theoretical limit. Overall, during the entire discharge process, the color of electrolyte turns from colorless to gray to dark yellow and back to light yellow, demonstrating that the content of soluble polysulfides in the electrolyte initially increases and then decreases. This result is in accordance with the tested sulfur content in the electrolyte at various discharge stages using ICP-OES in our previous work $[34,35]$.

The subsequent charge process from $\mathrm{Li}_{2} \mathrm{~S}$ to $\mathrm{S}_{8}$ was also studied using our in-operando cell design. A typical sloping charge potential profile was observed as shown in Figure $2 \mathrm{~b}$. We see that the color of the electrolyte become increasingly dark as the charging proceeds (in set of Figure $2 b$ and Figure S2), accompanied by an increase of the corresponding gray level (Figure 2d). The digital images also show a similar transition process (inset of Figure $2 \mathrm{~b}$ ). This result indicates increasing amount of soluble long chain polysulfides in the electrolyte during the charge process. This result is also consistent with the tested sulfur content in the electrolyte at various charge stages using ICP-OES in our previous work [34,35]. The maximum of long chain polysulfides coincides with the voltage rise, indicating the final oxidation of $\mathrm{S}_{8}{ }^{2-}$ to $\mathrm{S}_{8}$. The dark color in the electrolyte at the end of charge process indicates that some long chain soluble polysulfides remain in the electrolytes, confirming the incomplete conversion from $\mathrm{Li}_{2} \mathrm{~S}$ to $\mathrm{S}_{8}$ [36-38]. Overall, the schematic in Figure $\mathrm{S} 3$ summarizes the conversion of sulfur species both in the cathode and electrolyte during the charge/discharge processes. 
After studying the temporal distribution of soluble polysulfide species during the discharge/charge process, we next proceeded to examine their spatial distribution. In order to observe the spatial distribution, the colors and the corresponding grey levels in the electrolyte $v s$. the distance from the cathode to the anode were analysed. Figure 3a displays an optical image of the electrolyte in an as-designed in-operando lithium-sulfur cell at the DOD of 38.9\%. As mentioned above, the maximum concentration of soluble polysulfides in the electrolyte was found at this stage based on the observed gray level, making it the easiest point for visualization of their spatial distribution. As can be seen, the color of the electrolyte is brown-yellow. It is worth noting that there is a color gradient between the cathode side and the anode side (Figure 3a). The color near the sulfur cathode is darker than that near the lithium anode, which implies the diffusion of soluble polysulfides from the cathode to anode in the discharge process. Moreover, the corresponding grey levels (Figure 3b) clearly indicate the color gradient in the electrolyte, further validating the spatial distribution of soluble polysulfides. The diffusion of soluble polysulfide species from the cathode to the anode leads to the "shuttle" phenomenon, resulting in low Coulombic efficiency that plagues lithiumsulfur batteries [2-5]. In addition, the comparison of the color change and their grey levels in the electrolyte $v s$. the distance from the cathode to the anode at various DOD were carried out (Figure S4). Compared to the case at $\sim 38.9 \%$ DOD, the color gradient vs. distance in the electrolyte is much smaller at the initial and final stages of discharge (2.8\% and 100\% DOD) due to the lower contents of soluble polysulfide species. The spatial distribution of polysulfide species in the electrolyte $v s$. the distance from the cathode to the anode during the charge process was also investigated (at the DOC of $\sim 90 \%$ ). There also exists a color gradient during the charge process from the cathode side to the anode side as illustrated in Figure $3 \mathrm{c}$ and $\mathrm{d}$.

To suppress the diffusion of polysulfides, rational design of the cathode structure is important. In our previous work, well-defined, PEDOT-encapsulated hollow sulfur 
nanoparticles were prepared and they showed good long-term cycling stability compared to their unencapsulated counterparts [20]. Recently, it was also reported that a well-designed functional separator with Nafion coating can work as ionic shield to block the diffusion of these soluble polysulfides to the anode and localize them on the cathode side, leading to enhanced cycling performance [23]. In both cases, although the cycling performances are greatly improved, there is no direct visual evidence of polysulfide trapping. To observe this for the first time, in-operando lithium-sulfur cells were assembled by using pristine sulfur electrode, sulfur-PEDOT composite electrode and a Nafion modified separator, respectively. The distribution of polysulfides in the electrolyte for these in-operando cells in different DOD and DOC was visualized.

Figure 4 shows the optical images and their gray levels at various DOD and DOC during the first cycle for these tested in-operando lithium-sulfur cells. As shown in Figure 4a-c, characteristic potential plateaus of lithium-sulfur batteries are observed in the discharge/charge potential profiles of the three in-operando lithium-sufur cells using a normal separator, sulfur-PEDOT composite electrode and a Nafion modified separator, respectively. The electrode consisting of hollow sulfur nanoparticles with a PEDOT shell shows a short potential tail in the range of $1.8-1.7 \mathrm{~V}$ during the first discharge process (Figure 4b), different from that of micro-sized sulfur particles (Figure 4a), which may arise from the decomposition of $\mathrm{LiNO}_{3}$ in the electrolyte on the active surface of sulfur-PEDOT nanocomposites [39]. A discharge slope between 2.1-1.7 $\mathrm{V}$ and a charge slope between 2.4-2.6 V are observed for the lithium-sulfur cell using a Nafion modified separator (Figure 4c), in accordance with the previous results [23]. Optical images are selected and compared at the same DOD and DOC for these tested in-operando cells (Figure 4d). As discussed earlier, the in-operando cell with an unencapsulated sulfur cathode and an ordinary separator (Celgard 2300) exhibited a dark yellow color at the DOD of $\sim 38.9 \%$. In contrast, the electrolyte shows a much lighter color for 
the in-operando cell with a sulfur-PEDOT electrode, which indicates a lower degree of polysulfide dissolution into the electrolyte. This provides direct evidence of the effect of PEDOT coating on the confinement of polysulfides within the cathode, which helps us to understand the superior cycling life of sulfur-PEDOT composite cathode. Moreover, we also see that with the introduction of a Nafion coated separator, the color of electrolyte is light yellow at the DOD of $\sim 38.9 \%$, instead of the dark yellow color observed for the cell using an ordinary separator. These results support the claim that polysulfides are blocked at the cathode side by the functional separator with Nafion coating. The grey levels of all the measured in-operando lithium-sulfur cells are shown in Figure 4e. The cells with sulfurPEDOT composite cathode and a Nafion coated separator stay at a low level of grey level during the whole discharge/charge processes. Compared with the in-operando cell using an ordinary separator and a pristine sulfur cathode, they exhibit reduced color intensity variation during the discharge/charge cycle. Therefore, our in-situ observation provides direct evidence to the effective confinement of polysulfides in advanced lithium-sulfur cell designs with a PEDOT-coated sulfur electrode and a Nafion modified separator.

\section{Conclusions}

In summary, we have explored a simple in-operando lithium-sulfur cell design for direct visualization of the spatial and temporal distribution of soluble lithium polysulfides. The asmade in-operando lithium-sulfur cells were studied under real-time discharge/charge conditions, and the behavior of polysulfides were monitored under an optical microscope, which enabled us to clearly understand the loss of active mass and the "shuttle effect" in lithium-sulfur batteries, paving the way for their further improvements in electrochemical performances. Moreover, the present cell design was successfully applied to investigate the trapping of polysulfudes in lithium-sulfur cells with a sulfur-PEDOT composite cathode and a 
functional Nafion modified separator. Additionally, our in-operando cell is versatile for other in-situ characterizations (e.g., Raman spectra) and can also be applicable to other battery systems, which may be helpful for understanding their electrochemical properties.

\section{Acknowledgment}

Y.C. acknowledges the support from the Assistant Secretary for Energy Efficiency and Renewable Energy, Office of Vehicle Technologies of the U.S. Department of Energy.

\section{References}

[1] P. G. Bruce, S. A. Freunberger, L. J. Hardwick and J. M. Tarascon, Nat. Mater. 11 (2012) $19-29$.

[2] Y. Yang, G. Y. Zheng and Y. Cui, Chem. Soc. Rev. 42 (2013) 3018 - 3032.

[3] S. Evers and L. F. Nazar, Acc. Chem. Res. 46 (2012) 1135 - 1143.

[4] A. Manthiram, Y. Z. Fu and Y. S. Su, Acc. Chem. Res. 46 (2013) 1125 - 1134.

[5] Y. X. Yin, S. Xin, Y. G. Guo and L. J. Wan, Angew. Chem. Int. Ed. 52 (2013) 2 - 18.

[6] X. L. Ji, K. T. Lee and L. F. Nazar, Nat. Mater. 8 (2009) 500 - 506.

[7] Y. Yang, M. T. McDowell, A. Jackson, J. J. Cha, S. S. Hong and Y. Cui, Nano Lett. 10 (2010) $1486-1491$.

[8] S. Q. Chen, X. D. Huang, H. Liu, B. Sun, W. Yeoh, K. F. Li, J. Q. Zhang and G. X. Wang, Adv. Energy Mater. 130176 (2014).

[9] S. Xin, L. Gu, N.-H. Zhao, Y.-X. Yin, L.-J. Zhou, Y.-G. Guo and L.-J. Wan, J. Am. Chem. Soc. 134 (2012) 18510 - 18513.

[10] L. W. Ji, M. M. Rao, H. M. Zheng, L. Zhang, Y. C. Li, W. H. Duan, J. H. Guo, E. J. Cairns and Y. G. Zhang, J. Am. Chem. Soc. 133 (2011) 18522 - 18525. 
[11] H. L. Wang, Y. Yang, Y. Y. Liang, J. T. Robinson, Y. G. Li, A. Jackson, Y. Cui and H. J. Dai, Nano Lett. 11 (2011) 2644 - 2647.

[12] M.-Q. Zhao, Q. Zhang, J.-Q. Huang, G.-L. Tian, J.-Q. Nie, H.-J. Peng and F. Wei, Nat. Commun. 5 (2014) 3410.

[13] Y. Yang, G. H. Yu, J. J. Cha, H. Wu, M. Vosgueritchian, Y. Yao, Z. N. Bao and Y. Cui, Acs Nano 5 (2011) 9187 - 9193.

[14] L. F. Xiao, Y. L. Cao, J. Xiao, B. Schwenzer, M. H. Engelhard, L. V. Saraf, Z. M. Nie, G. J. Exarhos and J. Liu, Adv. Mater. 24 (2012) 1176 - 1181.

[15] X. L. Ji, S. Evers, R. Black and L. F. Nazar, Nat. Commun. 2 (2011) 325.

[16] G. Y. Zheng, Y. Yang, J. J. Cha, S. S. Hong and Y. Cui, Nano Lett. 11 (2011) 4462 4467.

[17] G. Y. Zheng, Q. F. Zhang, J. J. Cha, Y. Yang, W. Y. Li, Z. W. Seh and Y. Cui, Nano Lett. 13 (2013) $1265-1270$.

[18] Z. W. Seh, W. Y. Li, J. J. Cha, G. Y. Zheng, Y. Yang, M. T. McDowell, P.-C. Hsu and Y. Cui, Nat. Commun. 4 (2013) 1331.

[19] W. Y. Li, G. Y. Zheng, Y. Yang, Z. W. Seh, N. Liu and Y. Cui, Proc. Nat. Acad. Sci. 110 (2013) $7148-7153$.

[20] W. Y. Li, Q. F. Zhang, G. Y. Zheng, Z. W. Seh, H. B. Yao and Y. Cui, Nano Lett. 13 (2013) $5534-5540$.

[21] H. B. Yao, G. Y. Zheng, P.-C. Hsu, D. S. Kong, J. J. Cha, W. Y. Li, Z. W. Seh, M. T. McDowell, K. Yan, Z. Liang, V. K. Narasimhan and Y. Cui, Nat. Commun. 5 (2014) 3943.

[22] Y.-S. Su, A. Manthiram, Nat. Commun. 3 (2012) 1166.

[23] J.-Q. Huang, Q. Zhang, H.-J. Peng, X.-Y. Liu, W. Qian and F. Wei, Energy Environ. Sci. 7 (2013) $347-353$.

[24] G. M. Zhou, S. F. Pei, L. Li, D.-W. Wang, S. G. Wang, K. Huang, L.-C. Yin, F. Li and H.-M. Cheng, Adv. Mater. 26 (2013) 625 - 631.

[25] L. M. Suo, Y.-S. Hu, H. Li, M. Armand and L. Q. Chen, Nat. Commun. 4 (2013) 1481. [26] J.-T. Yeon, J.-Y. Jang, J.-G. Han, J. Cho, K. T. Lee and N.-S. Choi, J. Electrochem. Soc. 159 (2012) A1308 - A1314.

[27] Y. Diao, K. Xie, S. Z. Xiong and X. B. Hong, J. Electrochem. Soc. 159 (2012) A1816 A1821.

[28] D. Aurbach, E. Pollak, R. Elazari, G. Salitra, C. S. Kelley and J. Affinito, J. Electrochem. Soc. 156 (2009) A694 - A702. 
[29] M. Hagen, P. Schiffels, M. Hammer, S. Dörfler, J. Tübke, M. J. Hoffmann, H. Althues and S. Kaskel, J. Electrochem. Soc. 160 (2013) A1205 - A1214.

[30] J. Nelson, S. Misra, Y. Yang, A. Jackson, Y. Liu, H. L. Wang, H. J. Dai, J. C. Andrews, Y. Cui and M. F. Toney, J. Am. Chem. Soc. 134 (2012) 6337 - 6343.

[31] M. U. M. Patel, R. Demir-Cakan, M. Morcrette, J.-M. Tarascon, M. Gaberscek and R. Dominko, ChemSusChem 6 (2013) 1177 - 1181.

[32] M. Cuisinier, P.-E. Cabelguen, S. Evers, G. He, M. Kolbeck, A. Garsuch, T. Bolin, M. Balasubramanian and L. F. Nazar, J. Phys. Chem. Lett. 4 (2013) 3227 - 3232.

[33] N. A. Cañas, D. N. Fronczek, N. Wagner, A. Latz and K. A. Friedrich, J. Phys. Chem. C 118 (2014) $12106-12114$.

[34] Z. W. Seh, H. T. Wang, N. Liu, G. Y. Zheng, W. Y. Li, H. B. Yao and Y. Cui, Chem. Sci. 5 (2014) $1396-1400$.

[35] Z. W. Seh, H. T. Wang, P.-C. Hsu, Q. F. Zhang, W. Y. Li, G. Y. Zheng, H. B. Yao and Y. Cui, Energy Environ. Sci. 7 (2014) $672-676$.

[36] S.-E. Cheon, K.-S. Ko, J.-H. Cho, S.-W. Kim, E.-Y. Chin and H.-T. Kim, J.

Electrochem. Soc. 150 (2003) A800 - A805.

[37] H.-S. Ryu, H.-J. Ahn, K.-W. Kim, J.-H. Ahn and J.-Y. Lee, J. Power Sources 153 (2006) $360-364$.

[38] S. S. Jeong, Y. T. Lim, Y. J. Choi, G. B. Cho, K. W. Kim, H. J. Ahn and K. K. Cho, J. Power Sources 174 (2007) $745-750$.

[39] S. S. Zhang, J. Electrochem. Soc. 159 (2012) A920 - 923.

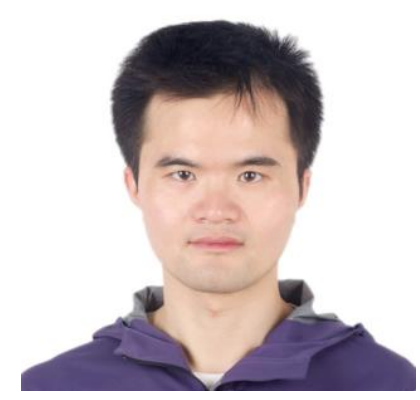

Yongming Sun received his Ph.D. degree from Huazhong University of Science and Technology (P. R. China) in 2012. He is currently working with Professor Yi Cui as a 
postdoctoral associate at Stanford University. His research focuses on nanomaterials for energy storage.

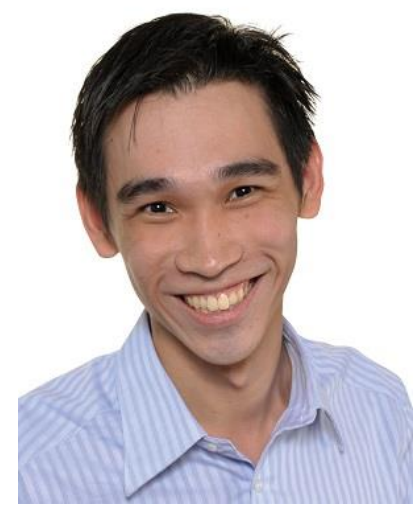

Zhi Wei Seh received his B.S. degree from Cornell University in 2010 and worked for a year at the Institute of Materials Research and Engineering, A*STAR in Singapore. He is currently pursuing a Ph.D. in Materials Science and Engineering at Stanford University under the A*STAR National Science Scholarship. His research focuses on nanostructured electrode materials for advanced $\mathrm{Li}$-ion and $\mathrm{Li}-\mathrm{S}$ batteries.

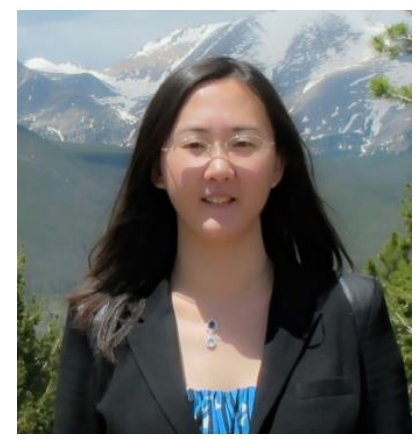

Weiyang Li graduated with B.S. (2004) and M.S. (2007) degrees in Chemistry from Nankai University (P. R. China), and a Ph.D. in Biomedical Engineering from Washington University in St. Louis (2011, with Prof. Younan Xia). She is now working with Prof. Yi Cui as a postdoctoral associate in the Department of Materials Science and Engineering at Stanford University. Her research focuses on the design and synthesis of novel nanostructured materials with controlled compositions, sizes, and shapes to address critical problems related to energy storage devices. 


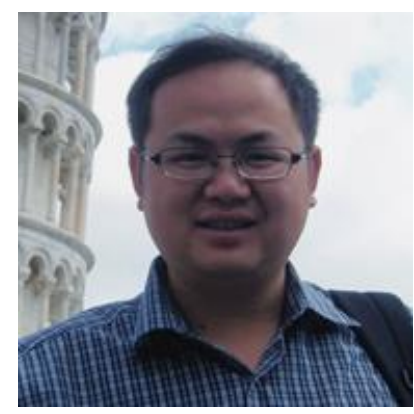

Hong-Bin Yao is a post-doctoral working with Professor Yi Cui at Stanford University. He received his B. S. degree in chemistry from University of Science and Technology of China (USTC) in 2006 and earned his PhD in chemistry with Professor Shu-Hong Yu at USTC in 2011. During 2007-2008, he attended the joint-training program for studying crystal structure analysis in Professor Jing Li's group at Rutgers University. His current research focuses on biotemplating nanostructured materials for energy storage and lithium sulfur battery.

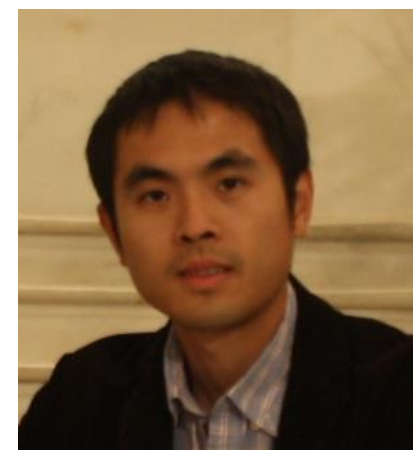

Guangyuan Zheng received his B.A. degree in Chemical Engineering from University of Cambridge in 2009. He obtained his PhD degree in Chemical Engineering from Stanford University in 2014, working on high performance lithium sulfur batteries and lithium metal anode. Currently he is doing postdoctoral research in the Department of Materials Science and Engineering at Stanford, co-supervised by Prof. Yi Cui and Prof. Steven Chu. His research focuses on the development of nanomaterials for energy storage applications. 


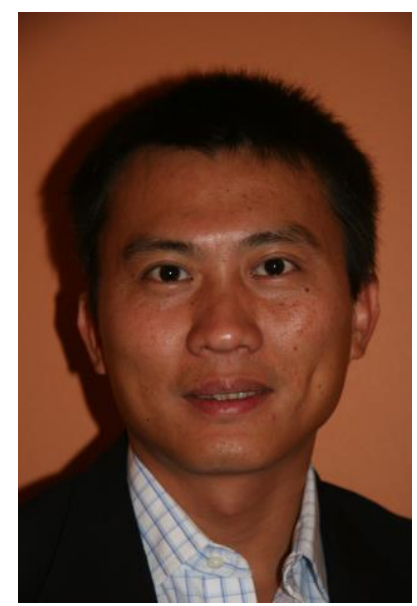

Yi Cui received his B.S. degree in chemistry from the University of Science and Technology of China in 1998 and his Ph.D. degree in chemistry from Harvard University in 2002. He went on to work as a Miller Postdoctoral Fellow at the University of California, Berkeley. In 2005, he became a professor in the Department of Materials Science and Engineering at Stanford University. He leads a group of researchers working on nanomaterials for energy, environment, electronics and biology. Among many honors, he has received the Inaugural Nano Energy Award (2014), Wilson Prize from Harvard University (2011), the KAUST Investigator Award (2008), the ONR Young Investigator Award (2008), the MDV Innovators Award (2007) and the Technology Review World Top Young Innovator Award (2004). 
Graphical Abstract
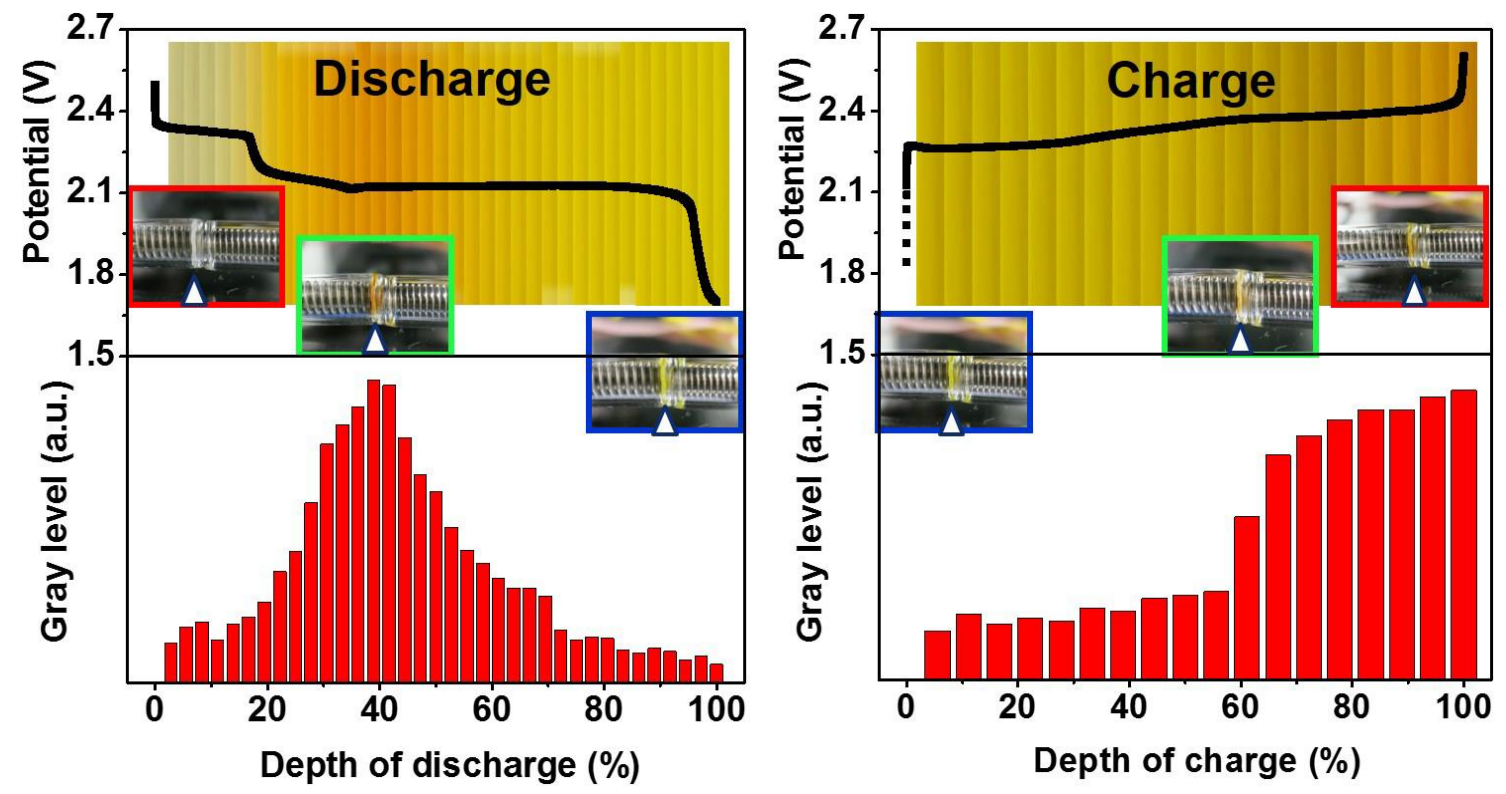


\section{Figure Captions:}

Figure 1 An in-operando lithium-sulfur cell design and its assembly: (a) schematic of an inoperando lihium-sulfur cell, (b) a glass tube used for housing the in-operando cell, (c) a stainless bolt before (left) and after (right) loading of a sulfur cathode, (d) a lithium metal counter electrode attached on a stainless bolt, (e) a Celgard 2300 separator (left) and a glass microfiber filter (right), (f) an assembled in-operando cell working under the optical microscope.

Figure 2 (a, b) The discharge and charge curves of the as-made in-operando lithium-sulfur cell at a current density of $10 \mu \mathrm{A}$ in the potential range of $1.7-2.6 \mathrm{~V}$ and (c, d) the corresponding change of gray level in the electrolyte with DOD and DOC. The inset digital images show the color change of the electrolyte in different DOD and DOC. The triangles show the positions of the glass microfibre filters in the in-operando cells.

Figure 3 The change of colors and the corresponding grey levels in the electrolyte vs. the distance from the cathode to the anode during the (a. b) discharge and (c, d) charge processes. The color gradient indicates the spatial distribution of soluble polysulfides from the cathode side to anode side. (e) The illustration of the spatial distribution of polysulfides in a lithiumsulfur battery.

Figure 4 The first discharge/charge curves of in-operando lithium-sulfur cells with (a) a normal sulfur electrode, (b) sulfur-PEDOT composite electrode, and (c) a Nafion modified separator, respectively. (d) Optical images and gray levels (e) of the measured in-operando lithium-sulfur cells at different DOD and DOC. The effect of encapsulation of sulfur with a PEDOT shell on the trapping polysulfide species was confirmed by our observation. The localization of polysulfides on the cathode side was also directly recorded when a Nafion modified separator was used. 


\section{Figures}

\section{Figure 1}

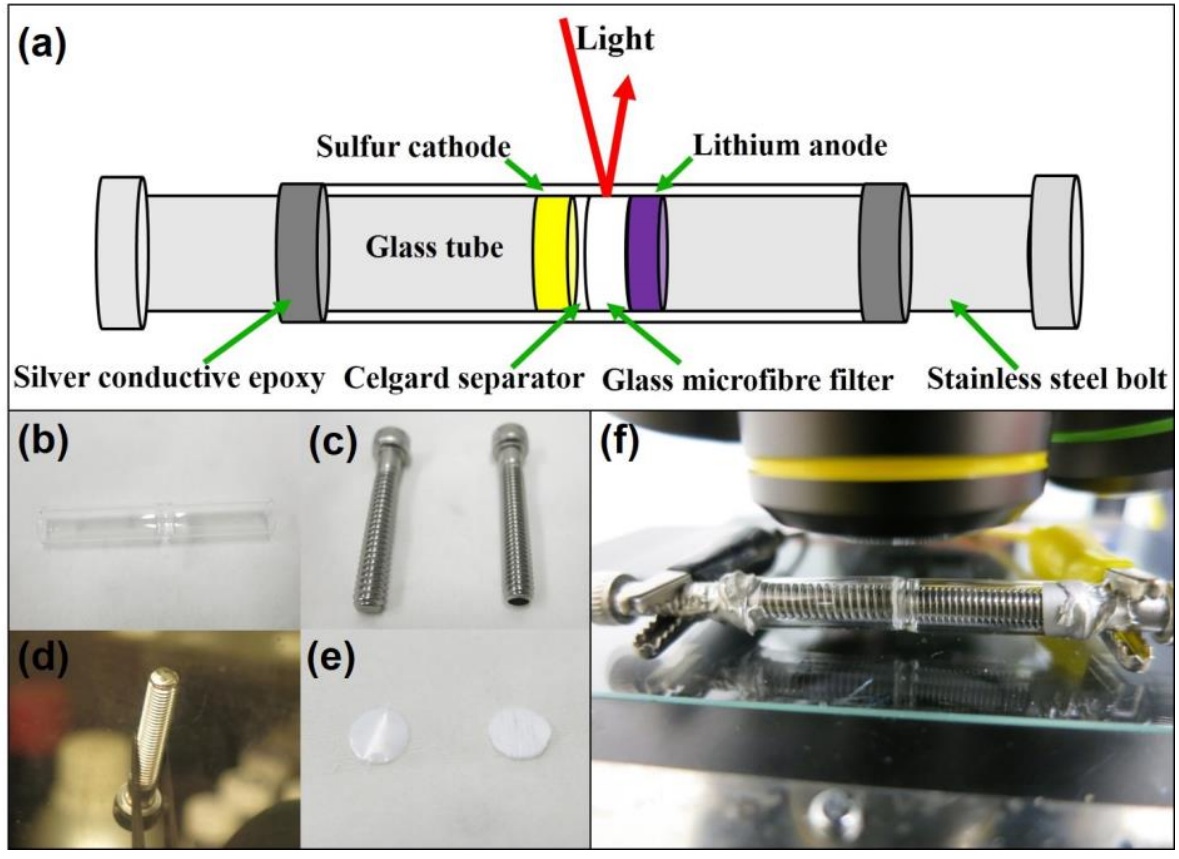


Figure 2
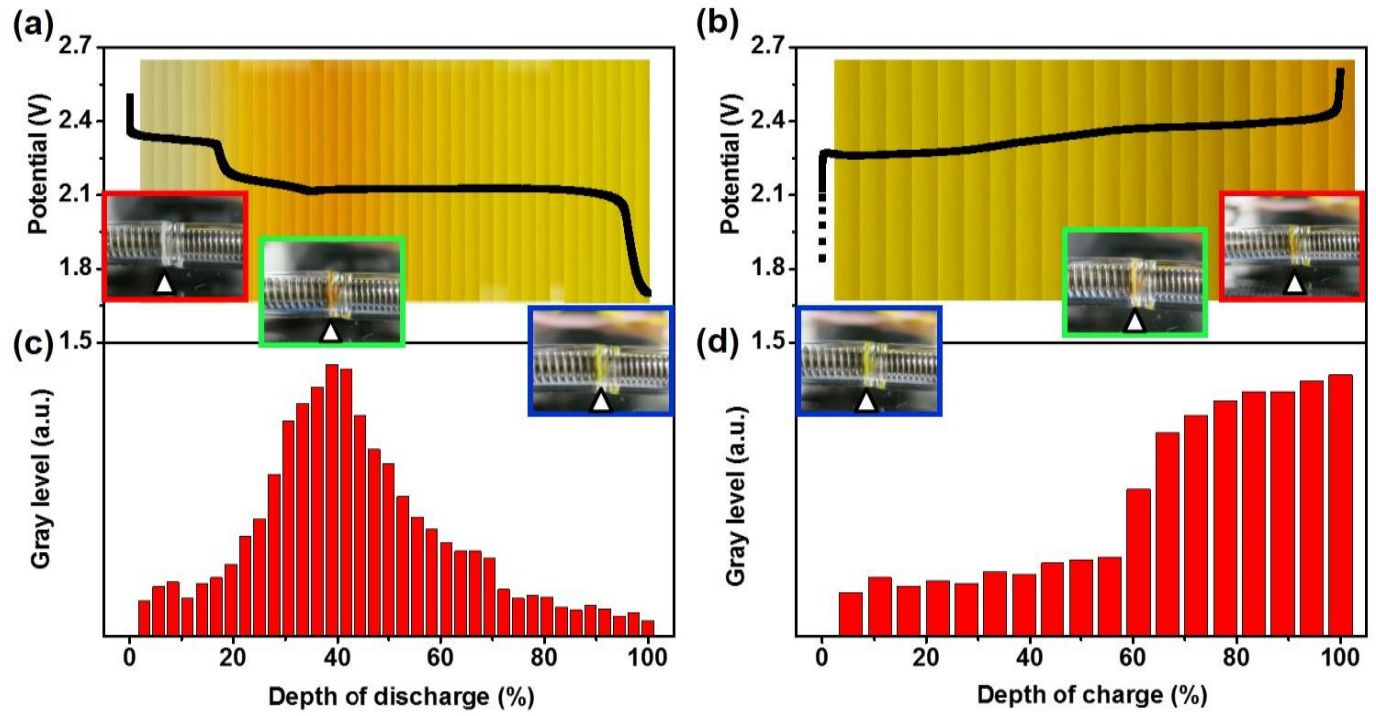
Figure 3

(a)

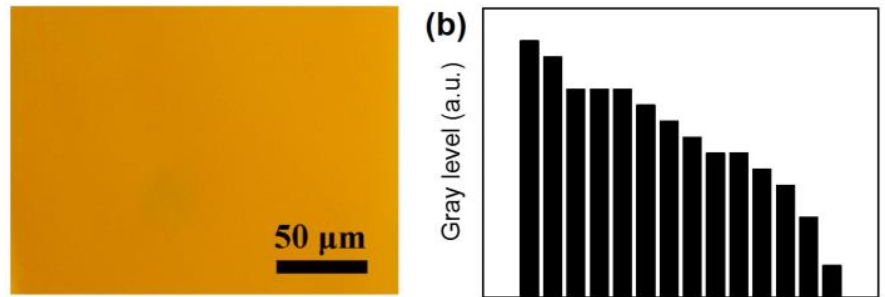

Cathode Anode Cathode

Position

(c)

Position
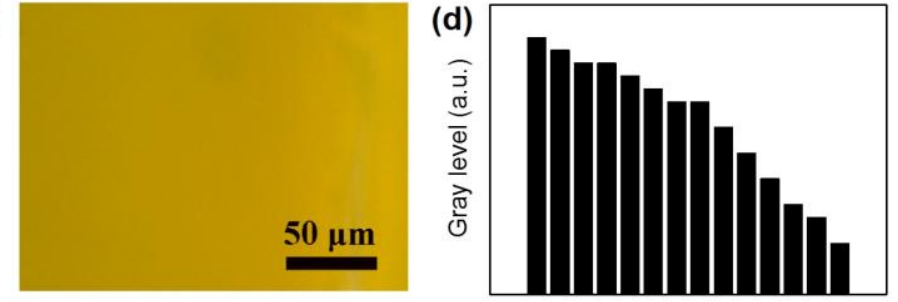

Cathode Anode Cathode Anode

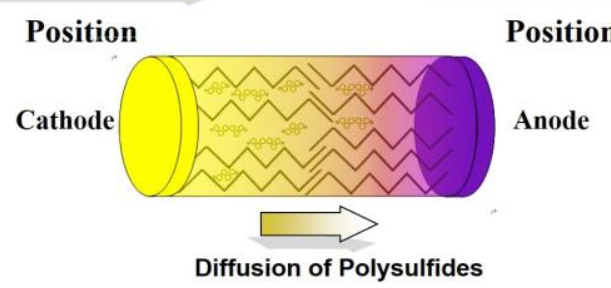

Diffusion of Polysulfides 
Figure 4
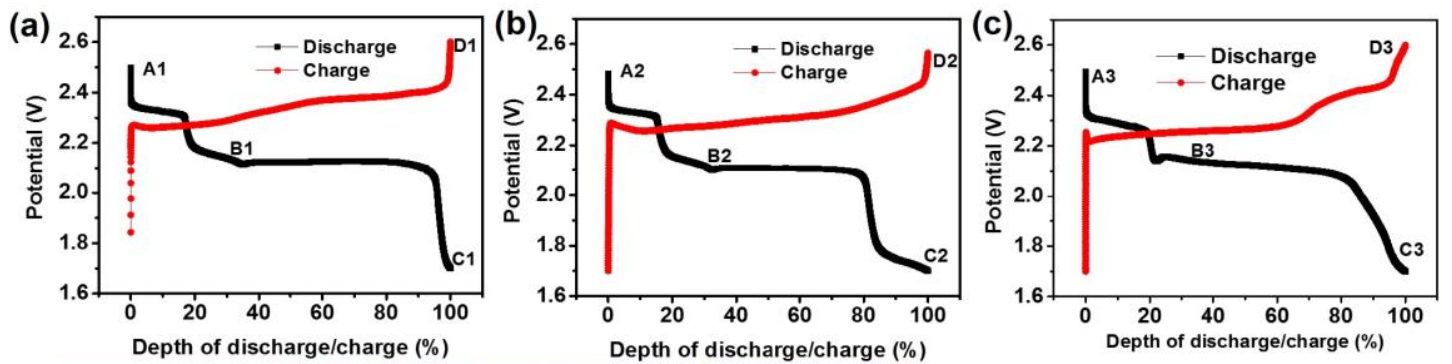

(d)

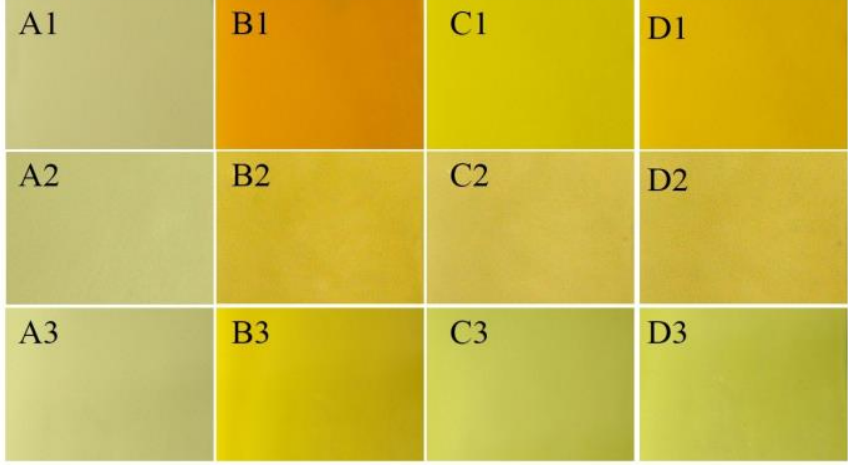

(e)

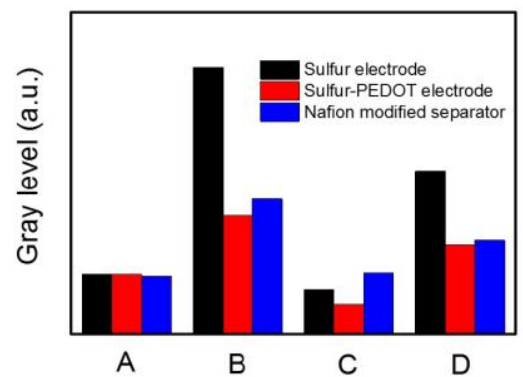

